\title{
Large-area molecular electronic devices from self-assembled monolayers
}

Kasper Nørgaard

Nano-Science Center \& Department of Chemistry, University of Copenhagen, Denmark E-Mail: $\underline{\text { nn } @ \text { nano.ku.dk }}$

A central goal in molecular electronics is to understand charge transport properties of single molecules and their ensembles. This fundamental knowledge may ultimately pave the way for electronic components constructed from tailor-made molecular building-blocks that can overcome some limits of conventional silicon-based microelectronics. The formation of robust and reproducible contact between molecule and electrode is a key challenge. At the single-molecule level, we have recently explored the use of parallel self-assembly strategies towards the formation of multiple single-molecule junctions, wherein the molecule-electrode interface is controlled with atomic precision [1]. However, the use of self-assembled monolayers (SAMs) offer a complementary approach for fabricating molecular electronic devices that are more amenable to device integration and up-scaling [2].

The presentation will highlight recent progress from our group on the fabrication and characterization of large-area molecular electronic devices employing chemically derived graphene as a soft contact-interface to the molecules. The graphene material serves a dual purpose in that it protects the underlying monolayer from filamentary damage when evaporating a metal top electrode, while itself functioning as a transparent, conducting electrode. The resulting solid-state devices are robust, permanent and can accommodate a diverse range of functional molecular layers, including e.g. photo-, redox- and bias-switches $[2,3]$, as well as a range of molecular binding groups. Further advancement in the chemical fabrication and processing of the graphene material has recently enabled reliable measurements of our devices at cryogenic temperatures and facilitated the measurement of inelastic electron tunneling spectroscopy (IETS) [4,5].

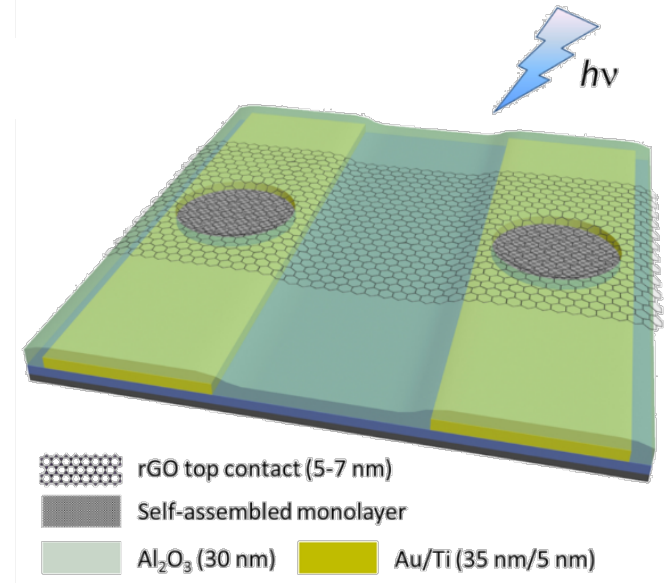

Figure 1: Schematic illustration of solid-state large-area molecular electronic device platform using a graphene material as a soft top-electrode for contacting the underlying molecular monolayer. 


\section{References.}

[1] T. Jain, Q. Tang, T. Bjørnholm and K. Nørgaard, Acc. Chem. Res., 47, 2-11 (2014).

[2] T. Li, M. Jevric, J.R. Hauptmann, R. Hviid, Z. Wei, R. Wang, N.E.A. Reeler, E. Thyrhaug, S. Petersen, J.A.S. Meyer, N. Bovet, T. Vosch, J. Nygård, X. Qiu, W. Hu, Y. Liu, G.C. Solomon, H.G. Kjaergaard, T. Bjørnholm, M.B. Nielsen, B.W. Laursen, K. Nørgaard, Adv. Mater., 25, 4164-4170 (2013).

[3] Z. Wang, H. Dong, T. Li, R. Hviid, Y. Zou, Z. Wei, X. Fu, E. Wang, Y. Zhen, K. Nørgaard, B.W. Laursen, W.P. Hu, Nature Commun., 6, 7478 (2015).

[4] M. Kühnel, M. H. Overgaard, M. C. Hels, A. Cui, T. Vosch, J. Nygård, T. Li, B. W. Laursen, K. Nørgaard, J. Phys. Chem. C, DOI: 10.1021/acs.jpcc.8b08377 (2018).

[5] M. Kühnel, S. V. Petersen, R. Hviid, M. H. Overgaard, B. W. Laursen, K. Nørgaard, J. Phys. Chem. C, 122 (18), 9731 (2018). 